\title{
Recurrence after operative management of intrahepatic cholangiocarcinoma
}

\author{
Omar Hyder, MD, MS, ${ }^{a}$ Ioannis Hatzaras, MD, ${ }^{a}$ Georgios C. Sotiropoulos, MD, ${ }^{b}$ Andreas Paul, MD, \\ Sorin Alexandrescu, MD, ${ }^{\mathrm{c}}$ Hugo Marques, MD, ${ }^{\mathrm{d}}$ Carlo Pulitano, MD, ${ }^{\mathrm{e}}$ Eduardo Barroso, MD, ${ }^{\mathrm{d}}$ \\ Bryan M. Clary, MD, ${ }^{\mathrm{f}}$ Luca Aldrighetti, MD, ${ }^{\mathrm{e}}$ Cristina R. Ferrone, MD, ${ }^{\mathrm{g}}$ Andrew X. Zhu, MD, PhD, ${ }^{\mathrm{g}}$ \\ Todd W. Bauer, MD, ${ }^{\mathrm{h}}$ Dustin M. Walters, MD, ${ }^{\mathrm{i}}$ Ryan Groeschl, MD, ${ }^{\mathrm{j}}$ T. Clark Gamblin, MD, MS, ${ }^{\mathbf{j}}$ \\ J. Wallis Marsh, MD, MBA, ${ }^{\mathrm{k}}$ Kevin T. Nguyen, MD, PhD, ${ }^{\mathrm{k}}$ Ryan Turley, MD, ${ }^{\mathrm{f}}$ Irinel Popescu, MD, \\ Catherine Hubert, MD, ${ }^{\mathrm{k}}$ Stephanie Meyer, MD, ${ }^{\mathrm{l}}$ Michael A. Choti, MD, ${ }^{\mathrm{a}}$ Jean-Francois Gigot, MD, ${ }^{\mathrm{k}}$ \\ Gilles Mentha, MD, ${ }^{1}$ and Timothy M. Pawlik, MD, MPH, PhD, ${ }^{\mathbf{a}}$ Baltimore, MD, Essen, Germany, \\ Bucharest, Romania, Lisbon, Portugal, Milan, Italy, Durham, NC, Boston, MA, Charlottesville, VA, Milwaukee, \\ WI, Pittsburgh, PA, Brussels, Belgium, and Geneva, Switzerland
}

\begin{abstract}
Introduction. Data on recurrence after operation for intrahepatic cholangiocarcinoma (ICC) are limited. We sought to investigate rates and patterns of recurrence in patients after operative intervention for ICC. Methods. We identified 301 patients who underwent operation for ICC between 1990 and 2011 from an international, multi-institutional database. Clinicopathologic data, recurrence patterns, and recurrencefree survival (RFS) were analyzed.

Results. During the median follow up duration of 31 months (range 1-208), 53.5\% developed a recurrence. Median RFS was 20.2 months and 5-year actuarial disease-free survival, $32.1 \%$. The most common site for initial recurrence after operation of ICC was intrahepatic ( $\mathrm{n}=98 ; 60.9 \%)$, followed by simultaneous intra- and extrahepatic disease $(\mathrm{n}=30 ; 18.6 \%) ; 33(21.0 \%)$ patients developed extrahepatic recurrence only as the first site of recurrence. Macrovascular invasion (hazard ratio [HR], 2.08; $95 \%$ confidence interval [CI], 1.34-3.21; $\mathrm{P}<.001)$, nodal metastasis (HR, 1.55; $95 \%$ CI, 1.01-2.45; $\mathrm{P}=.04)$, unknown nodal status $(H R, 1.57 ; 95 \%$ CI, $1.10-2.25 ; \mathrm{P}=.04)$, and tumor size $\geq 5 \mathrm{~cm}(H R$, 1.84; $95 \%$ CI, 1.28-2.65; $\mathrm{P}<.001)$ were independently associated with increased risk of recurrence. Patients were assigned a clinical score from 0 to 3 according to the presence of these risk factors. The 5-year RFS for patients with scores of 0, 1, 2, and 3 was $61.8 \%, 36.2 \%, 19.5 \%$, and 9.6\%, respectively. Conclusion. Recurrence after operative intervention for ICC was common. Disease recurred both at intraand extrahepatic sites with roughly the same frequency. Factors such as lymph node metastasis, tumor size, and vascular invasion predict highest risk of recurrence. (Surgery 2013;153:811-8.)
\end{abstract}

From the Department of Surgery, ${ }^{a}$ Johns Hopkins University School of Medicine, Baltimore, MD; Department of Surgery, ${ }^{b}$ University Hospital Essen, Essen, Germany; Institute for Digestive Diseases and Liver Transplantation Fundeni, ${ }^{c}$ Bucharest, Romania; Department of Surgery, ${ }^{d}$ Curry Cabral Hospital, Lisbon, Portugal; Department of Surgery, ${ }^{e}$ Ospedale San Raffaele, Milan, Italy; Department of Surgery, ${ }^{f}$ Duke Medical Center, Durham, NC; Department of Surgery, ${ }^{g}$ Massachusetts General Hospital, Boston, MA; Department of Surgery, ${ }^{h}$ University of Virginia, Charlottesville, VA; Department of Surgery, ${ }^{i}$ Medical College of Wisconsin, Milwaukee, WI; Department of Surgery, ${ }^{j}$ University of Pittsburgh, Pittsburgh, PA; Department of Surgery, ${ }^{k}$ Cliniques Universitaires Saint-Luc, Brussels, Belgium; and Department of Surgery, ${ }^{l}$ Hôpitaux Universitaires de Genève, Geneva, Switzerland

Presented at the 2012 Annual Society of Surgical Oncology Meeting, Orlando, FL.

Accepted for publication December 19, 2012.

Reprint requests: Timothy M. Pawlik, MD, MPH, PhD, Chief, Associate Professor of Surgery and Oncology, Division of Surgical Oncology, Department of Surgery, Blalock 688, 600 N. Wolfe Street, Baltimore, MD 21287. E-mail: tpawlik1@jhmi.edu.

0039-6060/\$ - see front matter

(C) 2013 Mosby, Inc. All rights reserved.

http://dx.doi.org/10.1016/j.surg.2012.12.005
INTRAHEPATIC CHOLANGIOCARCINOMA (ICG) is a relatively rare, malignant tumor located within the hepatic parenchyma. Despite its rarity, ICC is the second most common primary hepatic malignancy, after hepatocellular carcinoma, accounting for 5$30 \%$ of all primary liver cancer cases. In fact, the incidence of ICC in the Western hemisphere is rising; however, the reasons for this are poorly understood. ${ }^{1,2}$ Although many patients present with advanced and/or metastatic disease, a subset of 
patients have liver only disease that may be amenable to operative resection. Operative resection is the only potentially curative therapy for patients with ICC. ${ }^{3}$ Data from our group, as well as others, have noted a 5 -year survival after operative resection for ICC that ranges from 25 to $35 \%$. $^{4,5}$

Virtually all studies to date on outcomes after operative management of ICC have focused exclusively on overall survival. ${ }^{4,6-15}$ Specifically, previous investigators have reported on prognostic factors associated with worse overall survival. Data from our own international, multi-institutional ICC group have noted that higher tumor number, vascular invasion, and lymph node metastasis are associated with adverse survival outcomes. ${ }^{4}$ In contrast, data on recurrence, patterns of recurrence, and diseasefree survival remain ill-defined. As systemic and locoregional therapies emerge for the treatment of ICC, data on patterns of recurrence (eg, intra- vs extrahepatic) may help to inform treatment strategies. ${ }^{16-18}$ Unfortunately, the few studies that have reported recurrence data have been small (ie, $<100$ patients) and single center. ${ }^{5,19-22}$ As such, previous studies have been limited by sample size and an inability to generalize findings to other centers.

The aim of the current study was to investigate the incidence and patterns of recurrence after operative intervention for ICC. Specifically, we sought to examine which clinicopathologic factors were associated with recurrence after operative management of ICC.

\section{METHODS}

Between April 1990 and September 2011, 301 patients with nonmetastatic ICC treated primarily with curative intent operative resection were identified from 12 major hepatobiliary centers in the United States (The Johns Hopkins School of Medicine, Baltimore, MD; Duke University Medical Center, Durham, NC; University of Virginia, Charlottesville, VA; University of Pittsburgh Medical Center, Pittsburgh, PA; Massachusetts General Hospital, Boston, MA; Medical College of Wisconsin, Milwaukee, WI) and Europe (University Hospital Essen, Essen, Germany; Institute for Digestive Diseases and Liver Transplantation Fundeni, Bucharest, Romania; Curry Cabral Hospital, Lisbon, Portugal; Ospedale San Raffaele, Milan, Italy; Cliniques Universitaires Saint-Luc, Brussels, Belgium; Hôpitaux Universitaires de Genève, Geneva, Switzerland). The study was approved by the institutional review boards of the respective institutions. Only patients with histologically confirmed ICC who underwent initial treatment for ICC at a study center were included.
Data collection. As previously described, ${ }^{4} \mathrm{pa}-$ tients were evaluated with a baseline history and physical examination, serum laboratory tests, and appropriate imaging studies (eg, computed tomography or magnetic resonance imaging of the abdomen and pelvis and chest radiography or a chest computed tomography) at the discretion of the treating physician. Tumor characteristics were noted on imaging. Patients were considered potential surgical candidates if all disease was felt to be potentially resectable with curative intent with anticipated $\mathrm{R} 0$ resection and there was no evidence of extrahepatic disease. Data on treatment-related variables, such as type of operation, receipt of lymphadenectomy, and adjuvant therapy, were also obtained. Resection was classified as less than hemihepatectomy, hemihepatectomy, extended hepatectomy, or central hepatectomy. Margin and nodal status were ascertained based on final pathologic review by a dedicated hepatobiliary pathologist. The tumors were morphologically classified using the Liver Cancer Study Group of Japan criteria, and staged using the TNM classification. ${ }^{23,24}$ Patients with $\mathrm{Nx}$ disease were staged according to the T category status only. After surgery, all patients were regularly followed and prospectively monitored for recurrence with computed tomography or magnetic resonance imaging of the abdomen and a chest radiograph every 3-4 months up to 2 years, and then every 6 months thereafter until year 5, after which screening occurred annually.

Date of last follow-up, vital status, and recurrence-related information were collected for all patients. Recurrence was defined as a lesion that was biopsy-proven recurrent cholangiocarcinoma or a lesion that was deemed suspicious on crosssectional imaging in the setting of an elevated CA199 level. Information regarding the location, receipt of curative intent treatment for the recurrence, as well as the disease-free interval from the date of initial operation to the development of recurrent disease was recorded. Although the specific site of all recurrences were collected, for the purposes of analyses, recurrences were classified as intrahepatic only, extrahepatic only, or intra- and extrahepatic.

Statistical analyses. Summary statistics were obtained using established methods and presented as percentages, mean, or median values. Time to recurrence and survival were estimated using the Kaplan-Meier method and differences were compared using the log-rank test. Multivariate Cox regression analysis was performed to explore associations of factors with recurrence free survival. Hazard ratios and 95\% confidence intervals (CI) were estimated. Factors were entered into the 
multivariate models based on a univariate $P<.05$; multiple imputation was utilized to account for any missing data on specific factors. ${ }^{25,26}$ All statistical tests were 2-tailed. Statistical analyses were performed using SAS 9.3 (SAS Institute, Cary, NC).

\section{RESULTS}

Patient characteristics and overall survival. Table I lists the clinicopathologic features of the 301 patients in the study. There were 169 men $(56.2 \%)$ and 132 women $(43.9 \%)$. The median patient age was 64 years (range, 26-88). Most patients had a solitary tumor $(n=225 ; 74.8 \%)$, and the median tumor size was $7.2 \mathrm{~cm}$ (range, $0.7-21)$. A majority of patients had T1 category tumors $(n=175$; $58.1 \%)$; fewer had T2 $(n=66 ; 21.9)$ or T3/T4 tumors $(n=60 ; 20.0 \%)$. At the time of the operation, the extent of hepatic resection was less than a hemihepatectomy in $86(28.8 \%)$ patients, a hemihepatectomy in $115(38.1 \%)$ patients, and an extended hepatectomy in $100(33.1 \%)$ patients.

Most patients underwent R0 resection ( $n=244$; $81.1 \%)$; a smaller number had an $\mathrm{R} 1$ resection $(n=$ $57 ; 18.9 \%)$. Lymphadenectomy was performed in 182 patients $(60.5 \%) ; 50$ patients $(16.6 \%)$ had lymph node metastases. Patients who underwent lymphadenectomy were more likely to be younger (eg, $<60$ years), as well as have more advanced $\mathrm{T}$ category tumors (both $P<.05$ ). Vascular invasion was present in 94 patients: $39(13.0 \%)$ had microscopic invasion, and another 55 patients $(18.3 \%)$ had macrovascular invasion; 28 (9.3\%) patients had microscopic evidence of perineural invasion. In the postoperative setting, 109 (36.2\%) patients underwent adjuvant therapy; in the overwhelming majority of patients $(n=105 ; 95.4 \%)$ this consisted of adjuvant chemotherapy with or without adjuvant radiotherapy; the other 4 patients underwent adjuvant radiotherapy only.

Recurrence patterns and recurrence-free survival. During a median follow up of 31.0 months, most patients $(n=161 ; 53.5 \%)$ developed a recurrence. The most frequent site of initial recurrence after surgery of ICC was intrahepatic ( $n=98$; $60.9 \%$ ), followed by simultaneous intra- and extrahepatic disease $(n=30 ; 18.6 \%) ; 33(21.0 \%)$ patients developed extrahepatic only recurrence as the first site of recurrence.

Median overall survival was 37.8 months (95\% CI, 29.3-45.7). Median recurrence-free survival (RFS) was 20.2 months (95\% CI, 16.4-28.0). Overall 1-, 3-, and 5-year actuarial RFS was $64.2 \%$, $39.0 \%$, and $32.1 \%$, respectively (Fig 1 ).

Statistical analysis revealed several factors that influenced RFS. On univariate analysis, tumor size
Table I. Clinicopathologic features of 301 patients

\begin{tabular}{|c|c|}
\hline Variable & No. of patients (\%) \\
\hline \multicolumn{2}{|l|}{ Age (yrs) } \\
\hline$<60$ & $160(53.5)$ \\
\hline$\geq 60$ & $140(46.5)$ \\
\hline \multicolumn{2}{|l|}{ Gender } \\
\hline Female & $132(43.9)$ \\
\hline Male & $169(56.2)$ \\
\hline \multicolumn{2}{|l|}{ Tumor no. } \\
\hline Solitary & $225(74.8)$ \\
\hline Multiple & $76(25.3)$ \\
\hline \multicolumn{2}{|l|}{ Vascular invasion } \\
\hline Absent & $207(68.8)$ \\
\hline \multicolumn{2}{|l|}{ Present } \\
\hline Microscopic & $39(13.0)$ \\
\hline Major & $55(18.3)$ \\
\hline \multicolumn{2}{|l|}{ Node status } \\
\hline N1 & $50(16.6)$ \\
\hline No & $132(43.9)$ \\
\hline $\mathrm{Nx}$ & $119(39.5)$ \\
\hline \multicolumn{2}{|c|}{ American Joint Committee on Cancer $\mathrm{T}$ category } \\
\hline $\mathrm{T} 1$ & $175(58.1)$ \\
\hline $\mathrm{T} 2$ & $66(21.9)$ \\
\hline T3 & $53(17.6)$ \\
\hline $\mathrm{T} 4$ & $7(2.3)$ \\
\hline \multicolumn{2}{|l|}{ Type of operative resection } \\
\hline Less than hemihepatectomy & $86(28.6)$ \\
\hline Hemihepatectomy & $115(38.2)$ \\
\hline $\begin{array}{l}\text { Extended hepatectomy } \\
\quad(\geq 5 \text { liver segments })\end{array}$ & $100(33.2)$ \\
\hline
\end{tabular}

$\geq 5 \mathrm{~cm}$, nodal status, vascular invasion, multiple tumor nodules, and perineural invasion were all significant predictors of earlier recurrence. Patients with tumor size $\geq 5 \mathrm{~cm}$ had a median RFS of 17.0 months; in comparison patients with tumors $<5 \mathrm{~cm}$ had a median RFS of 41.2 months $(P<.001$; Fig 2, $A$ ). Patients with no lymph node metastasis (N0) had a median RFS of 35.1 months compared with 15.1 months for patients with lymph node metastasis (N1) and 15.6 months for node unknown lymph node status (Nx; $P=.01 ;$ Fig $2, B)$. Multiple tumors at time of operation were also associated with an earlier recurrence. Whereas patients with solitary tumors had a median RFS of 21.8 months, those with multiple tumors had a median RFS of 18.8 months $(P=.04)$. The presence of vascular invasion and perineural invasion was strong predictors of RFS (Fig 3). Among patients with no evidence of invasion, median RFS was 28.0 versus 18.1 months for patients with microscopic vascular invasion and 9.6 months for those with macrovascular invasion $(P$ $<.001$; Fig 3, A). Patients without perineural invasion had a median RFS of 21.8 months compared with 11.4 months for patients with perineural 


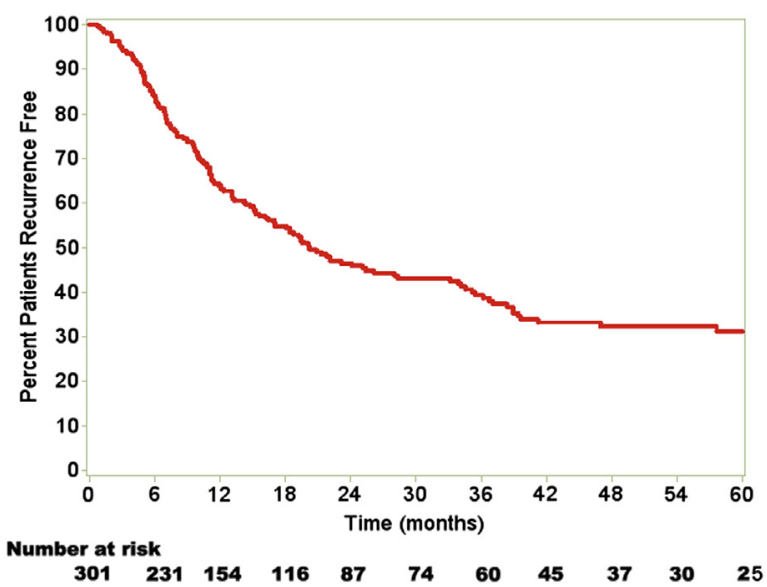

Fig 1. Median RFS was 20.2 months (95\% CI, 16.428.0). Overall 1-, 3-, and 5-year actuarial RFS was $64.2 \%, 39.0 \%$, and $32.1 \%$, respectively. (Color version of figure is available online.)

invasion $(P=.03$; Fig $3, B)$. Univariate analysis revealed no significant differences in RFS based on advanced age, extent of liver resection, receipt of adjuvant therapy, postoperative margin status, preoperative jaundice, presence of hepatic cirrhosis, presence of biliary invasion, or tumor histology (all $P>.05$ ).

On multivariable analysis, tumor size $\geq 5 \mathrm{~cm}$, macrovascular invasion, and lymph node status remained independent predictors of poor survival. Patients with tumors $\geq 5 \mathrm{~cm}$ had a greater likelihood of recurrence than those with smaller tumors (HR, 1.84; 95\% CI, 1.28-2.65; $P<.001)$. Patients with major vascular invasion had double the risk of recurrence compared with patients without vascular invasion (HR, 2.08; 95\% CI, 1.34-3.21, $P<.001$ ). In contrast, patients with no lymph nodes metastasis had a lower risk of recurrence compared with patient who had lymph node metastasis (HR, 1.55; $95 \% \mathrm{CI}, 1.01-2.45 ; P=.04)$. Other factors such as microvascular invasion, presence of multiple tumors, and perineural invasion were not independently associated with RFS (all $P>.05$; Table II).

Using the 3 independent variables-tumor size $\geq 5 \mathrm{~cm}$, major vascular invasion, and lymph node status-a clinical score was devised in an attempt to stratify patients with regard to prognosis. Patients were assigned a score based on the sum of these risk factors-patients lacking all 3 risk factors we assigned a score of 0 , patients with 1 risk factor were assigned a score of 1 , patients with 2 risk factors assigned a score of 2, and patients with 3 risk factors were assigned a score of 3 (Fig 4). An increasing risk score was associated with an incrementally worse RFS. Specifically, patients with a
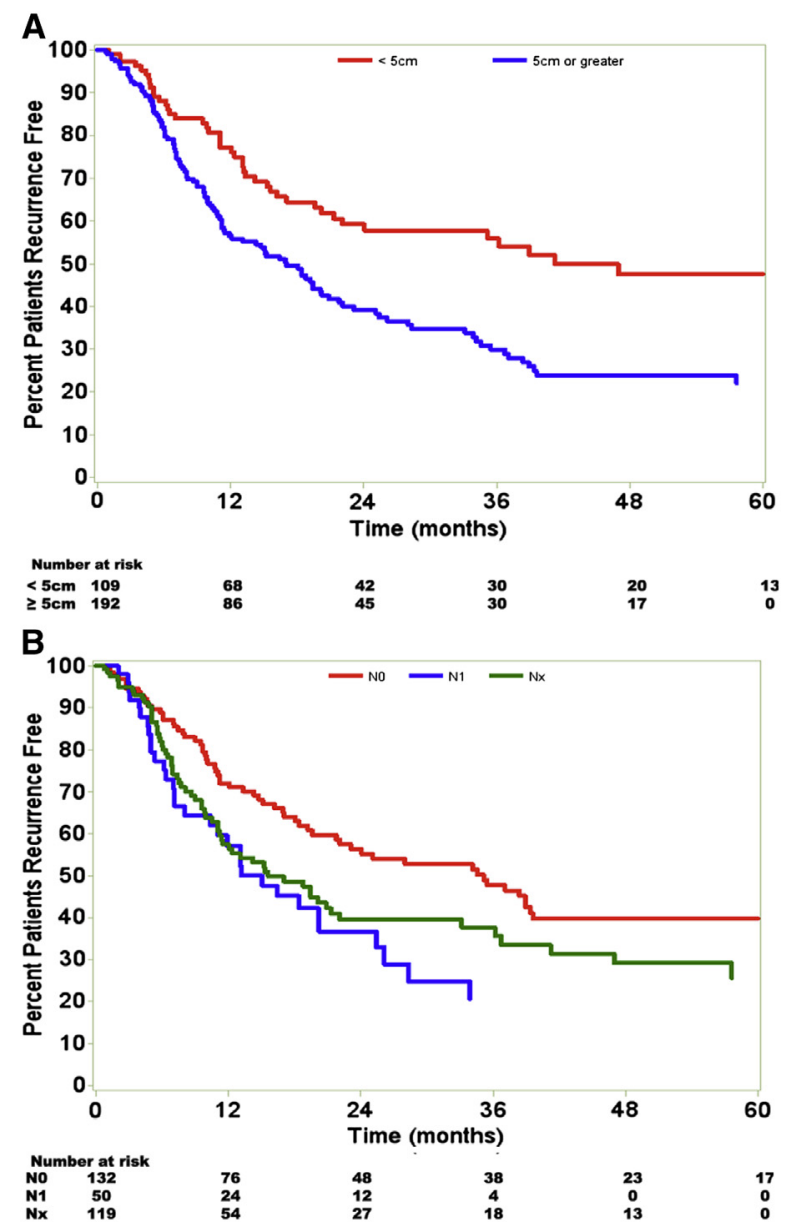

Fig 2. Tumor size and nodal status adversely affected the RFS. $A$, Patients with tumor size $\geq 5 \mathrm{~cm}$ had a worse RFS than those with smaller tumors. $B$, Patients with N0 disease also had a longer RFS than those with $\mathrm{N} 1$ and $\mathrm{Nx}$ disease. (Color version of figure is available online.)

score of 0 had a median RFS of 75.2 versus 34.6 months for a score of $1,11.1$ months for a score of 2 , and 9.0 months for a score of $3(P<.05$ for a score of 0 vs a score of 1 or 2 or 3 ). The 5 -year RFS for patients with scores $0,1,2$, and 3 was $61.8 \%, 36.2 \%, 19.5 \%$, and $9.6 \%$, respectively.

To assess our clinical scoring system, patients were also stratified by the American Joint Committee on Cancer TNM staging system, which is the generally accepted classification system used after resection. Under the TNM classification, patients with stage 1 disease had the longest median RFS (36.7 months) and better 5-year RFS rate $(38.8 \%)$ than patients with stage 2 or 3 disease $(P<.05$ for comparison of stage 1 with either stages 2 or 3 ). Patients with stages 2 and 3 disease had median RFS of 11.4 and 17.0 months, respectively. The 5-year RFS for stage 2 was $18.2 \%$, whereas patients with stage 3 disease had a corresponding RFS rate of 


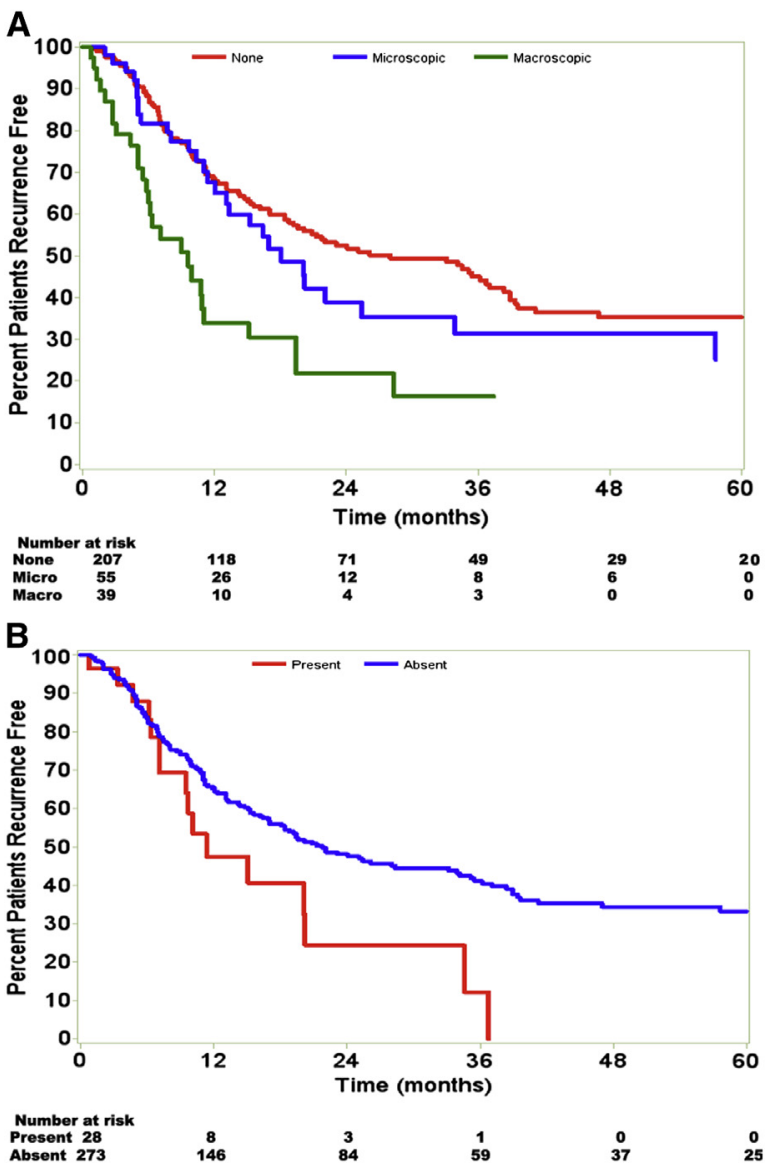

Fig 3. Vascular and perineural invasion both predicted poor survival. A, Patients with no vascular invasion, macroscopic, and microscopic invasion had a median RFS of 28.0, 9.6, and 18.1 months, respectively. $B$, Patients with perineural invasion had a median survival of 11.4 months compared with 21.8 months for patients without such invasion. (Color version of figure is available online.)

27.5\%. The relationship between the clinical score and the TNM stage is presented in Table III. The clinical score accurately identified most patients with early stage disease $-97.4 \%$ of patients with a score of 0 were TNM stage 1 or 2 . However, an advanced clinical score did not always correlate with an advanced TNM stage (ie, stage 3).

After recurrence at any site, median patient survival duration was 11.8 months (95\% CI, 8.014.1). Among the 161 patients who developed a recurrence, $33(20.5 \%)$ patients underwent a repeat curative intent operation. Among those patients who were treated operatively for recurrence with curative intent, the median survival was 25.8 months (95\% CI, 18.1-45.2), whereas those treated with systemic chemotherapy or best supportive care for the recurrence survived for a median duration of 9.2 months (95\% CI, 7.4-14.2; $P=.01$ ).

\section{DISCUSSION}

ICC is the second most common primary liver malignancy and the incidence of ICC is increasing worldwide. ${ }^{21}$ Operative therapy has been shown to be associated with survival in appropriately selected patients. ${ }^{4,20}$ As with virtually all solid tumors, the goal of operative intervention for ICC is complete extirpation of the disease to ensure the greatest chance of long-term cure. As such, most studies on operative therapy for ICC have focused almost exclusively on survival and those prognostic factors associated with overall survival. However, data on the incidence of recurrence and RFS are also important to patients and physicians. Although interested in the chance of cure and overall survival, patients also want information on the risk of recurrence. For physicians, data on recurrence and the specific patterns of recurrence can help to guide postoperative surveillance, as well as inform future therapeutic approaches. The current study is important because it defines the rate and pattern of recurrence after curative intent surgery for ICC in a large, multinational, multi-institutional cohort of patients. We found that more than one half of patients developed recurrence within 2 years. When patients initially recurred, the pattern of failure was distributed relatively equally among intraand extrahepatic disease.

The disease-free survival reported in the present study was notable for being $32.1 \%$ at 5 year with a median RFS of only 20.2 months. Previous smaller single-center studies had similarly high rates of recurrence with poor disease-free survival. ${ }^{19,22}$ However, unlike previous studies, we also defined the specific patterns of recurrence after curative intent surgery for ICC. Specifically, of the 161 patients who recurred, $60.9 \%$ patients recurred with liver only disease, whereas $39.1 \%$ developed a recurrence at an extrahepatic site with or without liver disease. Of note, the risk of both intra- and extrahepatic recurrence appeared to be highest over the first 24-36 months after surgery (Fig 1). Predictors of increased risk of recurrence included factors typically associated with poor tumor biology such as major vascular invasion and tumor size, among others. Interestingly, we had previously reported that tumor size was not associated with survival, whereas presence of multiple tumors was associated with overall survival. $^{8}$ In the current study, although the presence of multiple tumors was associated with RFS on univariate analyses, it was not on multivariate analyses. In addition, we noted that tumor size was associated with risk of recurrence, although it had not been associated with overall survival in our previously 
Table II. Factors used in formulating the clinical score

\begin{tabular}{|c|c|c|c|c|c|c|}
\hline \multirow[b]{2}{*}{ Parameter } & \multicolumn{4}{|c|}{ Recurrence-free survival } & \multirow{2}{*}{$\begin{array}{l}\text { Hazard ratio } \\
(95 \% \mathrm{CI})\end{array}$} & \multirow[b]{2}{*}{$\mathrm{P}$ value } \\
\hline & 1 year $(\%)$ & 3 year $(\%)$ & 5 year $(\%)$ & Median (mos) & & \\
\hline \multicolumn{7}{|l|}{ Vascular invasion } \\
\hline None & 68.8 & 44.8 & 35.2 & 28.0 & & \\
\hline Major vessel invaded & 34.2 & 16.2 & 16.2 & 9.6 & 2.08 & $<.001$ \\
\hline \multicolumn{7}{|l|}{ Tumor size $(\mathrm{cm})$} \\
\hline$<5$ & 77.4 & 55.8 & 47.6 & 41.2 & & \\
\hline$\geq 5$ & 56.2 & 30.0 & 22.0 & 17.0 & 1.84 & $<.001$ \\
\hline \multicolumn{7}{|l|}{ Lymph nodes status } \\
\hline No & 72.0 & 47.6 & 40.0 & 35.1 & & \\
\hline $\mathrm{N} 1$ & 59.8 & 20.4 & 20.4 & 15.1 & 1.55 & .04 \\
\hline $\mathrm{Nx}$ & 57.4 & 38.0 & 25.6 & 15.6 & 1.57 & .04 \\
\hline
\end{tabular}

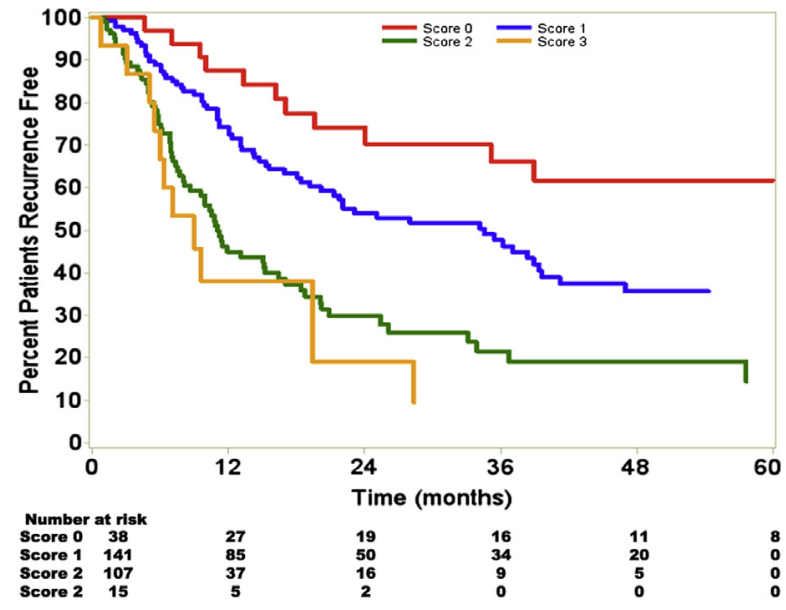

Fig 4. RFS declined with an increasing clinical score. The 5-year RFS for patients with scores $0,1,2$, and 3 was $61.8 \%, 36.2 \%, 19.5 \%$, and $9.6 \%$, respectively. (Color version of figure is available online.)

reported analyses. ${ }^{8}$ The reason for these discrepancies is probably multifactorial. Others have similarly noted that factors associated with recurrence do not necessarily correlate with overall survival. ${ }^{27,28}$ Although certain factors may predict initial risk of recurrence, the subsequent clinical course and natural history of the recurrence, as well as the progression of the disease leading to death, may be related to other prognostic factors more indicative of underlying disease biology such as vascular invasion and nodal status-both of which we previously reported were the main determinants of survival. Future research needs to continue to refine the prognostic factors associated with both recurrence-free and overall survival.

Recently, there has been increasing interest in the locoregional treatment of ICC not only operatively, but also with intra-arterial therapy ${ }^{29}$ or radiotherapy. ${ }^{30}$ To help better understand the
Table III. Relationship between clinical scoring system and American Joint Committee on Cancer TNM stage

\begin{tabular}{|c|c|c|c|c|}
\hline \multirow{2}{*}{$\begin{array}{c}\text { Clinical } \\
\text { score }\end{array}$} & \multirow{2}{*}{$\begin{array}{c}\text { Total no. } \\
\text { patients }\end{array}$} & \multicolumn{3}{|c|}{ No. (\%) of patients } \\
\hline & & Stage 1 & Stage 2 & Stage 3 \\
\hline 0 & 38 & $26(68.4)$ & $11(29.0)$ & $1(2.6)$ \\
\hline 1 & 141 & $89(63.1)$ & $20(14.2)$ & 32 (22.7) \\
\hline 2 & 53 & 37 (34.6) & $17(15.9)$ & $53(49.5)$ \\
\hline 3 & 15 & $2(13.3)$ & $5(33.3)$ & $8(53.3)$ \\
\hline
\end{tabular}

potential that local therapy might be able to provide for ICC, a better understanding of the natural history and recurrence of ICC is needed. The present study expands on our previous work and examines data on recurrence, RFS, and patterns of recurrence. We noted that at 5 years disease-free survival was only $32.1 \%$. Collectively, our data suggest that many patients treated with locoregional therapy not only recur, but recur rapidly and at a site outside their liver. As such, these data help to highlight the need to incorporate systemic therapy into future studies involving locoregional treatments for ICC.

Slightly more than one third of these patients underwent adjuvant therapy, most often consisting of systemic chemotherapy. In the current series, the use of adjuvant therapy was not associated with a decreased risk of recurrence. The reason for this is probably multifactorial. Given the respective nature of the current trial, there was undoubtedly some selection bias regarding who received adjuvant therapy. In addition, given that only one third of patients underwent adjuvant therapy, the smaller number of patients in this subset may have precluded our ability to detect an effect. In general, the use of adjuvant therapy for cholangiocarcinoma remains controversial and poorly 
defined. Factors predictive of recurrence, and the clinical prediction score herein proposed, may aid not only in prognostication of patients with ICC but also identify those patients at greatest risk of recurrence. In turn, such a risk score may assist in the selection of patients for receipt of adjuvant chemotherapy, because the subset of patients who should undergo such therapy remains unclear.

We demonstrated that disease-free survival mirrored overall survival, suggesting a high case fatality once recurrence occurs. Our finding that median survival was about 12 months among patients who recurred further supports this notion; in addition, this median survival was comparable with recently published prospective data on patients with advanced disease. Specifically, Valle et $\mathrm{al}^{16}$ reported on 410 patients with locally advanced or metastatic cholangiocarcinoma, gallbladder cancer, or ampullary cancer to receive either cisplatin followed by gemcitabine or gemcitabine alone. The authors reported that after a median follow-up of 8.2 months, the median overall survival was 11.7 months among the 204 patients in the cisplatin-gemcitabine group and 8.1 months among the 206 patients in the gemcitabine group (hazard ratio, $0.64 ; 95 \%$ CI, $0.52-0.80 ; P<$ $.001) .{ }^{16}$ As such, cisplatin plus gemcitabine has generally been accepted as the appropriate option for the treatment of patients with advanced biliary cancer. Whether this regimen will have a defined role in the adjuvant setting-and for what subset of patients-remains to be determined.

The current study had limitations that should be considered. Because our study is retrospective in nature, there may have been selection/detection bias. Similar to previous reports from our group, although all centers queried institutionwide databases for information on recurrence, it is conceivable that the extent of extrahepatic recurrences may be underrepresented owing to the surgical nature of the databases utilized in the current study. ${ }^{31}$ These limitations are inherent, however, in nearly all retrospective analyses of data on recurrence. The small number of patients in some subsets (eg, $<20 \%$ of cohort had an R1 margin) may have resulted in a type II error in assessing the difference between some potential prognostic factors. Finally, the absence of any centers from Asia/Eastern centers is another limitation of the current study. ICG findings based exclusively on a Western experience may not necessarily be applicable to ICC in the East. As such, future studies will not only need to validate the current clinical risk score, but also need to include data from Asian centers.
In conclusion, although 5-year survival after curative intent surgery for ICC was $32.1 \%$, recurrence after resection was common. In fact, more than half of patients recurred by 3 years and the 5year actuarial risk of both intra- and extrahepatic recurrence was $>60 \%$. The site of recurrence was relatively equally distributed with disease recurring both in the liver and at extrahepatic sites. Factors associated with recurrence included vascular invasion, tumor size, and lymph node metastasis. Data from the current study suggest that cure after resection of ICC remains an elusive goal. Future efforts needs to be aimed at better understanding the carcinogenesis and pathogenesis of ICC to identify more effective systemic therapeutic options for patients with this aggressive malignancy.

\section{REFERENCES}

1. Kaczynski J, Hansson G, Wallerstedt S. Incidence, etiologic aspects and clinicopathologic features in intrahepatic cholangiocellular carcinoma-a study of 51 cases from a lowendemicity area. Acta Oncol 1998;37:77-83.

2. Shaib Y, El-Serag HB. The epidemiology of cholangiocarcinoma. Semin Liver Dis 2004;24:115-25.

3. Benson AB 3rd, Abrams TA, Ben-Josef E, et al. NCCN clinical practice guidelines in oncology: hepatobiliary cancers. J Natl Comprehensive Cancer Network 2009;7:350-91.

4. de Jong MC, Nathan H, Sotiropoulos GC, et al. Intrahepatic cholangiocarcinoma: an international multi-institutional analysis of prognostic factors and lymph node assessment. J Clin Oncol 2011;29:3140-5.

5. Nuzzo G, Giuliante F, Ardito F, et al. Intrahepatic cholangiocarcinoma: prognostic factors after liver resection. Updates Surg 2010;62:11-9.

6. Isaji S, Kawarada Y, Taoka H, et al. Clinicopathological features and outcome of hepatic resection for intrahepatic cholangiocarcinoma in Japan. J Hepatobil Pancreatic Sur 1999;6:108-16.

7. Nakagohri T, Asano T, Kinoshita H, et al. Aggressive surgical resection for hilar-invasive and peripheral intrahepatic cholangiocarcinoma. World J Surg 2003;27:289-93.

8. Nathan H, Pawlik TM, Wolfgang CL, et al. Trends in survival after surgery for cholangiocarcinoma: a 30-year populationbased SEER database analysis. J Gastrointest Surg 2007;11: 1488-96.

9. Guglielmi A, Ruzzenente A, Campagnaro T, et al. Intrahepatic cholangiocarcinoma: prognostic factors after surgical resection. World J Surg 2009;33:1247-54.

10. Lang H, Sotiropoulos GC, Sgourakis G, et al. Operations for intrahepatic cholangiocarcinoma: single-institution experience of 158 patients. J Am Coll Surg 2009;208:218-28.

11. Nathan H, Aloia TA, Vauthey JN, et al. A proposed staging system for intrahepatic cholangiocarcinoma. Ann Surg Oncol 2009;16:14-22.

12. Hatzaras I, Schmidt C, Muscarella P, et al. Elevated CA 19-9 portends poor prognosis in patients undergoing resection of biliary malignancies. HPB 2010;12:134-8.

13. Nathan H, Pawlik TM. Staging of intrahepatic cholangiocarcinoma. Curr Opin Gastroenterol 2010;26:269-73.

14. Murakami Y, Uemura K, Sudo T, et al. Prognostic factors after surgical resection for intrahepatic, hilar, and distal cholangiocarcinoma. Ann Surg Oncol 2011;18:651-8. 
15. Poultsides GA, Zhu AX, Choti MA, Pawlik TM. Intrahepatic cholangiocarcinoma. Surg Clin North Am 2010;90:817-37.

16. Valle J, Wasan H, Palmer DH, et al. Cisplatin plus gemcitabine versus gemcitabine for biliary tract cancer. N Engl J Med 2010;362:1273-81.

17. Hong K, Geschwind JF. Locoregional intra-arterial therapies for unresectable intrahepatic cholangiocarcinoma. Semin Oncol 2010;37:110-7.

18. Zeng ZC, Tang ZY, Fan J, et al. Consideration of the role of radiotherapy for unresectable intrahepatic cholangiocarcinoma: a retrospective analysis of 75 patients. Cancer J 2006;12:113-22.

19. Chen LP, Li C, Wang C, et al. Predictive factors of recurrence for patients with intrahepatic cholangiocarcinoma after hepatectomy. Hepatogastroenterology 2012;59:1765-8.

20. Hanazaki K, Kajikawa S, Shimozawa N, et al. Prognostic factors of intrahepatic cholangiocarcinoma after hepatic resection: univariate and multivariate analysis. Hepatogastroenterology 2002;49:311-6.

21. Endo I, Gonen M, Yopp AC, et al. Intrahepatic cholangiocarcinoma: rising frequency, improved survival, and determinants of outcome after resection. Ann Surg 2008;248: 84-96.

22. Miwa S, Miyagawa S, Kobayashi A, et al. Predictive factors for intrahepatic cholangiocarcinoma recurrence in the liver following surgery. J Gastroenterol 2006;41:893-900.

23. Japan TLCSGo. Intrahepatic cholangiocarcinoma. In: Classification of primary liver cancer. Tokyo: Kanehara; 1997. p. 6-7.
24. Sobin LH, Wittekind CH, editors. TNM classification of malignant tumours. 6th edition Hoboken, NJ: Wiley; 2002.

25. Graham JW, Cumsille PE, Elek-Fisk E. Methods for handling missing data. In: Schinka JA, Velicer WF, editors. Research methods in psychology. New York: John Wiley \& Sons; 2003. p. 87-114.

26. Graham JW, Hofer SM. Multiple imputation in multivariate research. In: Little TD, editor. Modeling longitudinal and multiple-group data: practical issues, applied approaches, and specific examples. Hillsdale, NJ: Erlbaum; 2000. p. 201-18.

27. Raut CP, Tseng JF, Sun CC, et al. Impact of resection status on pattern of failure and survival after pancreaticoduodenectomy for pancreatic adenocarcinoma. Ann Surg 2007; 246:52-60.

28. de Haas RJ, Wicherts DA, Flores E, et al. R1 resection by necessity for colorectal liver metastases: Is it still a contraindication to surgery? Ann Surg 2008;248:626-37.

29. Park SY, Kim JH, Yoon HJ, et al. Transarterial chemoembolization versus supportive therapy in the palliative treatment of unresectable intrahepatic cholangiocarcinoma. Clin Radiol 2011;66:322-8.

30. Ibarra RA, Rojas D, Snyder L, et al. Multicenter results of stereotactic body radiotherapy (SBRT) for non-resectable primary liver tumors. Acta Oncol 2012;51:575-83.

31. de Jong MC, Pulitano C, Ribero D, et al. Rates and patterns of recurrence following curative intent surgery for colorectal liver metastasis: an international multi-institutional analysis of 1669 patients. Ann Surg 2009;250:440-8. 